\title{
Atomic motions in poly(vinyl methyl ether): A combined study by quasielastic neutron scattering and molecular dynamics simulations in the light of the mode coupling theory
}

\author{
S. Capponi, ${ }^{1,2, a)}$ A. Arbe ${ }^{3}$ F. Alvarez, ${ }^{2,3}$ J. Colmenero, ${ }^{1,2,3}$ B. Frick, ${ }^{4}$ and J. P. Embs ${ }^{5}$ \\ ${ }^{1}$ Donostia International Physics Center, Paseo Manuel de Lardizabal 4, 20018 San Sebastián, Spain \\ ${ }^{2}$ Departamento de Física de Materiales, UPV/EHU, Apartado 1072, 20080 San Sebastián, Spain \\ ${ }^{3}$ Centro de Física de Materiales (CSIC-UPV/EHU)-Materials Physics Center (MPC), \\ Apartado 1072, 20080 San Sebastián, Spain \\ ${ }^{4}$ Institut Laue-Langevin, BP 156, 38042 Grenoble Cedex 9, France \\ ${ }^{5}$ Laboratory for Neutron Scattering, ETH Zurich and PSI, CH-5232 Villigen PSI, \\ Switzerland and Physical Chemistry, Saarland University, D-66123 Saarbrücken, Germany
}

(Received 28 July 2009; accepted 14 October 2009; published online 23 November 2009)

\begin{abstract}
Quasielastic neutron scattering experiments (time-of-flight, neutron spin echo, and backscattering) on protonated poly(vinyl methyl ether) (PVME) have revealed the hydrogen dynamics above the glass-transition temperature. Fully atomistic molecular dynamics simulations properly validated with the neutron scattering results have allowed further characterization of the atomic motions accessing the correlation functions directly in real space. Deviations from Gaussian behavior are found in the high-momentum transfer range, which are compatible with the predictions of mode coupling theory (MCT). We have applied the MCT phenomenological version to the self-correlation functions of PVME atoms calculated from our simulation data, obtaining consistent results. The unusually large value found for the $\lambda$-exponent parameter is close to that recently reported for polybutadiene and simple polymer models with intramolecular barriers. (C) 2009 American Institute of Physics. [doi:10.1063/1.3258857]
\end{abstract}

\section{INTRODUCTION}

During the past decades, the study of the glass-transition has arisen as a great theoretical endeavor, the most significant success being the mode coupling theory (MCT) ${ }^{1,2}$ In addition, an enormous experimental effort has been made to characterize the dynamics in the supercooled regime of many systems. Some of these experiments aimed to a phenomenological characterization of the dynamical processes, mainly of the $\alpha$-relaxation (the process in which freezing leads to the glassy state), while others tried to check theoretical predictions. Among many other techniques, neutron scattering (NS) has been widely applied in this field. NS is extremely valuable due to its space-time resolution at a molecular level; in addition, it provides a direct check for the predictions of the MCT, since it allows accessing the dynamic structure factor. However, the application of MCT to the glasstransition of real systems is not trivial. ${ }^{3}$ In its original form, the MCT was formulated for simple liquids and it was numerically solved for rather simple systems as hard ${ }^{4,5}$ and soft $^{6}$ spheres, a binary Lennard-Jones mixture, ${ }^{7}$ and simple aspherical molecules with an intramolecular orientational degree of freedom. ${ }^{8-11}$ Experimental checks by NS have been performed with model ("canonical") systems as simple as possible, such as, e.g., the molecular system $o$-terphenyl. ${ }^{12}$ In general, the results support MCT predictions. Another family of good candidates are chemically simple polymers, such as the well-known 1,4-polybutadiene $(1,4-\mathrm{PB}),{ }^{13}$ that do not

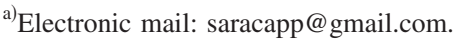

show a tendency to crystallize. Though 1,4-PB NS results were in qualitative accordance with MCT, the applicability of this theory to the glass-transition of a polymer melt, where connectivity and internal degrees of freedom play major roles, remained as an open question.

Later, molecular dynamics (MD)-simulations of a beadspring-type coarse-grained polymer model ${ }^{14}$ could be consistently analyzed in terms of the predictions of MCT, motivating an extension of this theory ${ }^{15}$ to reproduce the results of such model polymer simulations. Regarding more realistic polymer models, MCT has been applied to united-atom simulated polyethyelene ${ }^{16}$ and $1,4-\mathrm{PB} .{ }^{17}$ In the case of 1,4PB, a fully atomistic simulated cell, properly validated by direct comparison with NS results, has also been investigated in this line. ${ }^{18}$ The predictions seem to be fulfilled in all cases, though high values of the exponent parameters are obtained as compared to those found for the bead-spring models. ${ }^{14}$ The importance of the intramolecular barriers has been invoked to explain this effect. ${ }^{18-20}$ In any case, the questions of the applicability of the theory to real polymer systems and the origin of the unusual values found for the critical exponents are still open.

Poly(vinyl methyl ether) (PVME) was one of the first polymers for which supercooled dynamics was investigated by NS techniques. ${ }^{21-23}$ In those works, a phenomenological approach was followed to characterize the hydrogen motions, and no check of MCT was tried. The studies revealed a Gaussian behavior of the scattering function. Some years later, the deviations from Gaussian functional form of the scattering function of glass-forming systems in general 
spurred an enormous interest in the scientific community in connection with the question of the dynamic heterogeneities (see, e.g., Refs. 14 and 24-27). Seeking for these deviations, hints could also be found in the high- $Q$ range of the old PVME data, ${ }^{28}$ as well as in the case of other polymers. ${ }^{29-33}$ To shed light on the origin of such deviations, the information provided by MD-simulations is extremely useful, since it allows exploring relatively wide $Q$ and time windows (as compared with NS techniques) and accessing correlation functions directly in real space. Nevertheless, the realism of the simulated cell has to be properly verified, and a synergetic combination of both means (NS on real samples and MD-simulations) is the most advisable tool to address these questions, as it has recently been proven in several systems. $^{31,33-38}$

In this work we have applied such a combined approach to the study of PVME atomic motions in the supercooled regime. New experiments using three different spectrometers on a protonated sample have provided valuable additional insight on the H-dynamics. By time-of-flight (ToF), we have observed a two-step decay of the intermediate scattering function around the picosecond region. This technique has also allowed determining the $Q$-dependence of this function in the second decay at high- $Q$ and temperature. In addition, with neutron spin echo (NSE) and backscattering we have extended the previously explored $Q$-range toward lower $Q$-values (below $0.4 \AA^{-1}$ ) and lower temperatures. The NS data have also been used to validate our fully atomistic MDsimulations on PVME. The phenomenological analysis of the slow decay of the scattering function obtained from both sets of data shows clear deviations from Gaussian behavior at high $Q$. Interestingly enough, the way these deviations manifest is well compatible with the predictions of the MCT. This finding, together with other hints for the consistency of the MCT provided by our data (two-step relaxation functions, evidences of cage effect in real space), has motivated an analysis of PVME MD-simulations results in the light of the phenomenological version of the MCT. In this way, with this work we also contribute to the question about the applicability of this theory to real (or realistic) polymers.

\section{EXPERIMENTAL}

\section{A. Samples}

PVME (chemical formula $\left[-\mathrm{CH}_{2}-\mathrm{CHO}\left(\mathrm{CH}_{3}\right)_{-}\right]_{n}$ ) was purchased from Sigma-Aldrich. The glass-transition temperature $T_{g}$, measured through a differential scanning calorimetry measurement, was found to be $247.1 \mathrm{~K}$, in accordance with literature data. ${ }^{39}$ Before filling the flat aluminum holders used for NS experiments, the sample was carefully dried out in a vacuum oven. The sample thickness $(0.2 \mathrm{~mm})$ was chosen to provide a transmission of about $90 \%$, thus allowing multiple scattering effects to be neglected.

\section{B. Quasielastic NS}

In a typical NS experiment, the measured intensity as a function of energy $\hbar \omega$ and momentum transfer $\hbar Q$ provides information on the dynamics and structure of the sample under investigation. The modulus of the momentum transfer
$Q$ is determined by the scattering angle $\theta$ and the wavelength of the incoming neutrons $\lambda$ as $Q=4 \pi \sin (\theta / 2) / \lambda$. The measured intensity contains incoherent and coherent contributions that are weighted according to the corresponding cross sections $\left(\sigma_{\text {inc }}, \sigma_{\text {coh }}\right)$. The incoherent scattering function $S_{\text {inc }}(Q, \omega)$ is the Fourier transform of the intermediate incoherent scattering function $S_{\text {inc }}(Q, t)$ and the double Fourier transform of $S_{\text {inc }}(Q, \omega)$ yields the self-part of the van Hove correlation function $G_{\text {self }}(r, t)$,

$$
G_{\text {self }}(r, t)=\frac{1}{N_{\alpha}}\left\langle\sum_{i=1}^{N_{\alpha}} \delta\left[r-\left|\vec{r}_{i}(t)-\vec{r}_{i}(0)\right|\right]\right\rangle,
$$

where $N_{\alpha}$ is the number of nuclei of the considered kind of isotope $\alpha$ in the sample. In the classical limit, $G_{\text {self }}(r, t)$ is the probability of a given nucleus to be at distance $r$ from the position where it was located at a time $t$ before $\left(\vec{r}_{i}\right.$ denotes the position of the particle $i$ and the brackets mean ensemble average). Incoherent scattering looks at correlations among the positions of the same nucleus at different times.

On the other hand, coherent scattering deals with relative positions of atomic pairs (see, as general references, Refs. 40-43). The NS cross sections for the different atoms involved in the chemical formula of PVME are: $\sigma_{\text {inc }}^{\mathrm{H}}$ $=79.7 \mathrm{~b} ; \sigma_{\text {coh }}^{\mathrm{H}}=1.76 \mathrm{~b} ; \sigma_{\text {inc }}^{\mathrm{C}}=0 ; \sigma_{\text {coh }}^{\mathrm{C}}=5.56 \mathrm{~b} ; \sigma_{\text {inc }}^{\mathrm{O}}=0$; and $\sigma_{\text {coh }}^{\mathrm{O}}=4.23 \mathrm{~b}$. Therefore, the intensity scattered by the sample is dominated by the scattering from hydrogens, and thereby provides information about the self-motion of such atoms, $S_{\text {inc }}^{\mathrm{H}}(Q, \omega)$.

In the following, we introduce some definitions related with the functional form of the self-part of the van Hove correlation function that will be invoked along the paper. For some simple cases-free nuclei in a gas, harmonic crystals, simple diffusion at long times- $G_{\text {self }}(r, t)$ is a Gaussian function; ${ }^{40,42}$ in an isotropic system this implies

$$
G_{\text {self }}^{\text {gauss }}(r, t)=\left[\frac{\alpha(t)}{\pi}\right]^{3 / 2} \exp \left[-\alpha(t) r^{2}\right] .
$$

The even moments of $G_{\text {self }}(r, t)$

$$
\left\langle r^{2 n}\right\rangle=\int_{0}^{\infty} r^{2 n} G_{\text {self }}(r, t) 4 \pi r^{2} d r
$$

can be straightforwardly calculated in the Gaussian approximation. For instance, the mean squared displacement of the atom $\left\langle r^{2}(t)\right\rangle$ is given by $\left\langle r^{2}(t)\right\rangle=3 /[2 \alpha(t)]$. Moreover, in such Gaussian case, $S_{\text {inc }}(Q, t)$ is entirely determined by $\left\langle r^{2}(t)\right\rangle$

$$
S_{\text {inc }}^{\text {gauss }}(Q, t)=\exp \left[-\frac{\left\langle r^{2}(t)\right\rangle}{6} Q^{2}\right] \text {. }
$$

In more general cases, deviations of $G_{\text {self }}(r, t)$ from the Gaussian form [Eqs. (2) and (4)] may be expected. $S_{\text {inc }}(Q, t)$ can then be expressed in terms of its expansion in $Q^{2}$ (see, e.g., Refs. 44 and 45),

$$
S_{\text {inc }}(Q, t)=\exp \left[-\frac{\left\langle r^{2}(t)\right\rangle}{6} Q^{2}+\frac{\alpha_{2}(t)\left\langle r^{2}(t)\right\rangle^{2}}{72} Q^{4}+\cdots\right],
$$

where $\alpha_{2}(t)$, giving the leading correction, is the so-called second order non-Gaussian parameter. It can be obtained 
from the second $\left(\left\langle r^{2}(t)\right\rangle\right)$ and fourth $\left(\left\langle r^{4}(t)\right\rangle\right)$ moments of $G_{\text {self }}(r, t)$,

$$
\alpha_{2}(t)=\frac{3}{5} \frac{\left\langle r^{4}(t)\right\rangle}{\left\langle r^{2}(t)\right\rangle^{2}}-1 .
$$

For a perfect Gaussian curve, $\alpha_{2}(t)=0$.

\section{Time of flight}

In the microscopic time region $(\approx 0.2-30 \mathrm{ps})$, the self motions of PVME protons were investigated by ToF techniques. ToF measurements yield the energy-resolved scattered intensity at different angles. Properly taking into account the change in the $Q$-vector due to the change in neutron energy at each scattering angle, the quasielastic spectra at given constant $Q$-values can be obtained.

In this work we used the spectrometer FOCUS at the spallation source SINQ (Paul Scherrer Institut, Villigen). In FOCUS, the $375{ }^{3} \mathrm{He}$ counter tubes in three banks detect the scattered neutrons after passing a $2.5 \mathrm{~m}$ flight distance filled with argon. Thereby, the scattering angles ranged between $10^{\circ}$ and $130^{\circ}$. Using and incident wavelength of $\lambda=5 \AA$, the energy resolution of the experiment was $\delta E$ (HWHM) $\approx 45 \mu \mathrm{eV}$. We focused on the temperature of $400 \mathrm{~K}$. The resolution was determined from a measurement of the sample at very low temperature $(20 \mathrm{~K})$, where the scattering is only elastic. Background corrections were performed by subtracting the intensity scattered by the empty cell. The detector efficiency was corrected by the measurement of a standard vanadium sample. Measuring times of the order of $5 \mathrm{~h}$ were employed. After interpolating the signal at constant $Q$ in a reasonable energy transfer region, the effective $Q$-range was reduced to values between 0.62 and $2.21 \AA^{-1}$.

\section{Neutron spin echo}

NSE is distinguished from all other dynamic NS techniques in that it measures directly the velocity change in each neutron individually during scattering. ${ }^{46-48}$ This allows a decoupling of the neutron bandwidth utilized by the instrument from the obtainable resolution. The velocity measurements are performed using the Larmor precession angle of the neutron spin in a magnetic field. A comparison of these Larmor angles before and after scattering leads to a direct measurement of velocity differences, irrespective of the initial velocity of neutrons.

A singular feature of NSE is that it gives information in Fourier space and measures the time-dependent correlation functions. The time variable depends on the precession field $B$, the wavelength $\lambda$, and the length of the precession field $L$ $t \sim B L \lambda^{3}$. These experiments finally access the normalized function

$$
\operatorname{NSE}(Q, t)=\frac{\sigma_{\mathrm{coh}} S_{\mathrm{coh}}(Q, t)-\frac{1}{3} \sigma_{\mathrm{inc}} S_{\mathrm{inc}}(Q, t)}{\sigma_{\mathrm{coh}} S_{\mathrm{coh}}(Q, 0)-\frac{1}{3} \sigma_{\mathrm{inc}} S_{\mathrm{inc}}(Q, 0)},
$$

which has both coherent and incoherent contributions. Due to the polarization analysis involved, the incoherent contribution to the NSE signal is reduced to $1 / 3$ of the total incoherent intensity. For this reason, NSE is usually employed to investigate samples that scatter mainly coherently. However, in our protonated sample, the incoherent component is still strong enough as compared to the coherent one to dominate the NSE signal. The measurements on PVME thus directly reveal $S_{\text {inc }}(Q, t)$ related to the self-motion of the hydrogens. Moreover, deconvolution from the resolution function can be easily performed by simple division of the results by those obtained from a sample that scatters elastically. This provides valuable information on the spectral shape that is difficult to obtain by means of other NS techniques (and in a wider dynamic window).

The experiments were carried out by means of the NSE instrument IN11C at the Institute Laue-Langevin (ILL) in Grenoble (France). ${ }^{47}$ The multidetector at IN11C covers an angular range of $30^{\circ}$ in the horizontal plane. The selector provides a monochromatization of $\Delta \lambda / \lambda=15 \%$. With an incident wavelength $\lambda=5.5 \AA$, the multidetector was placed at $25^{\circ}$ scattering angle $\theta$ for its central detector. Thereby, a $Q$-range $0.25 \leq Q \leq 0.72 \AA^{-1}$ and a time range $5.3 \mathrm{ps} \leq t$ $\leq 0.95 \mathrm{~ns}$ were explored. The measurements were performed at 375 and $400 \mathrm{~K}$. Typical measuring times were $2.5 \mathrm{~h}$. The instrumental resolution function was determined from the elastic scattering of the sample at $10 \mathrm{~K}$. The instrumental background from the sample environment and the $\mathrm{Al}$ container was measured with a similar measuring time and thereafter subtracted from the experimental spectra using the appropriate transmission factor. The instrumental resolution effects were finally removed by dividing the background corrected spectra by the resolution function.

\section{Backscattering}

Backscattering spectrometers work under indirect geometry condition, i.e., the energy of the detected neutrons is fixed to a given value $E_{f}$, while the energy of the incident neutrons is varied around $E_{f}$. To operate, these instruments can use ToF or not. In the latter case, perfect crystals act as monochromator and analyzers and the instrumental resolution is optimized by using backscattering geometry at both crystals. ${ }^{49}$ This is the basis of the IN16 spectrometer ${ }^{50}$ at the ILL used in this work. With a wavelength of $6.271 \AA$, IN16 offers an energy resolution of nearly Gaussian shape and covers a $Q$-range between 0.19 and $1.9 \AA^{-1}$. We investigated three temperatures above $T_{g}, 325,350$, and $400 \mathrm{~K}$, employing measuring times of about $8 \mathrm{~h}$. The resolution function of the spectrometer was determined from the measurement of a sample at $2 \mathrm{~K}$. The acquired data were corrected for detector efficiency, sample container, and absorption using the standard programs available at ILL, thus finally providing the experimental scattering function.

\section{MD-simulations}

Fully atomistic MD-simulations were carried out using the COMPASS forcefield implemented within the commercial software package MATERIALS STUDIO 4.1. Based on the polymer consistent forcefield (PCFF), the COMPASS was developed using a hybrid approach consisting of $a b$ initio parametrization and empirical optimization method. The former method was used to derive the forcefield parameters, while the latter yielded a good agreement between calcula- 
tion and experiments. The forcefield functional forms are characterized by two different kind of functions: the valence terms, which include diagonal and off-diagonal crosscoupling terms, and the nonbond interaction terms. The valence terms represent internal coordinates of bond, angle, torsion angle, and out-of-plane angle; the cross-coupling terms include combinations of two or three internal coordinates. The nonbond interactions include a Lennard-Jones 9-6 function for the van der Waals terms and a Coulombic function for electrostatic interactions. Details of the analytical expression of the functional form are given in Refs. 51-53.

The initial configuration of the simulated system was built by means of the MATERIALS STUDIO 4.1 amorphous cell builder. ${ }^{54}$ A cubic cell containing seven PVME chains of 100 monomers was constructed at $400 \mathrm{~K}$, under periodic boundary conditions. By means of $N P T$ dynamic runs (i.e., keeping the number of atoms, pressure, and temperature constant), a value of the density of $\rho=0.981 \mathrm{~g} / \mathrm{cm}^{3}$ was determined. This value is in excellent agreement with the literature data. ${ }^{55}$ Such a density leads to a cell dimension of $40.986 \AA$. In order to minimize the obtained energy structure, the Polak-Ribiere conjugated gradients method was used. After the minimization, the system was dynamically equilibrated by a successive NVT run (i.e., keeping the number of atoms, volume, and temperature constant) of $1 \mathrm{~ns}$. The system obtained in such way was used as a starting point for collecting data every $0.01 \mathrm{ps}$ during a run of $1 \mathrm{~ns}$. The simulations were carried out in the NVT ensemble and the velocity-Verlet algorithm with a time step of $1 \mathrm{fs}$ was used as the integration method. To control the temperature, instead of a real temperature-bath coupling (Nosé-Hoover or Berendsen thermostat) a velocity scaling procedure with a wide temperature window of $10 \mathrm{~K}$ was followed. Under these conditions, greater temperature fluctuations are allowed but the trajectory is disturbed less. It has been checked that, by following this simple procedure, the results are similar to those obtained with an NVE ensemble (where the number of atoms, volume, and energy are kept constant), which has the proper Newtonian dynamics. ${ }^{56}$ After the first 1 ns run, successive runs of $20 \mathrm{~ns}$ collecting data every $0.5 \mathrm{ps}$ were carried out in order to reach a $40 \mathrm{~ns}$ dynamics. This same cell was used to yield simulated systems at the lower temperatures of 375 , 350,325 , and $300 \mathrm{~K}$. The new cells were obtained by scaling the temperature and were then equilibrated following the same procedure of the starting one. Once the equilibrium density was reached for each temperature, the same protocol of the first simulated sample was followed to carry out a $40 \mathrm{~ns}$ dynamics. The densities of the equilibrated cells were found to be $\rho(375 \mathrm{~K})=0.996 \mathrm{~g} / \mathrm{cm}^{3}, \quad \rho(350 \mathrm{~K})$ $=1.015 \mathrm{~g} / \mathrm{cm}^{3}, \quad \rho(325 \mathrm{~K})=1.033 \mathrm{~g} / \mathrm{cm}^{3}$, and $\rho(300 \mathrm{~K})$ $=1.046 \mathrm{~g} / \mathrm{cm}^{3}$.

\section{RESULTS AND VALIDATION}

\section{A. NS}

Figure 1(a) shows the FOCUS results for four of the $Q$-values investigated at $400 \mathrm{~K}$. Due to the influence of the instrumental resolution and the contributions of the fast dynamical processes (below $\approx 2 \mathrm{ps}$ ), ${ }^{57}$ it is difficult to deter-

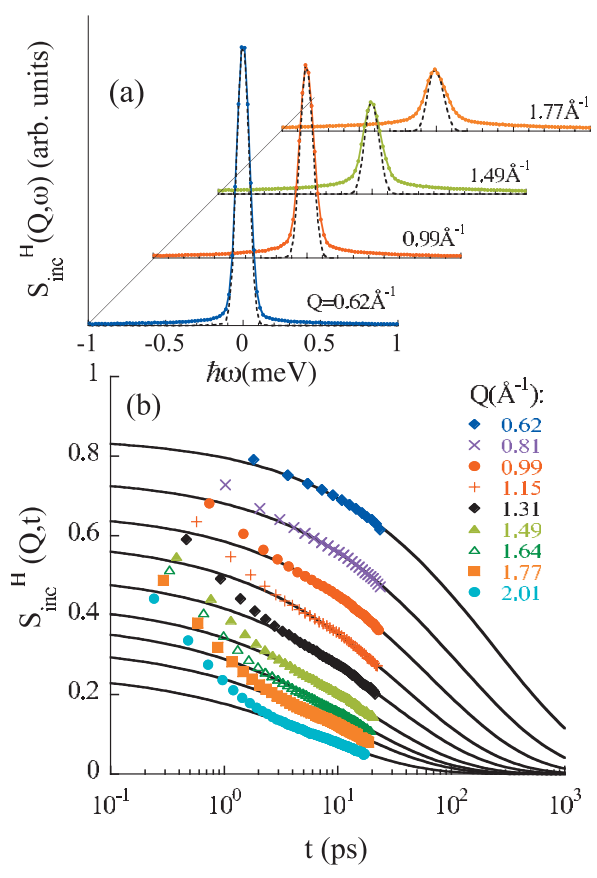

FIG. 1. (a) Spectra obtained with FOCUS at $T=400 \mathrm{~K}$ at the indicated $Q$-values. The dashed lines show the resolution function. (b) Fourier transformed data deconvoluted with the instrumental resolution. The solid lines are KWW fits of the second step.

mine the exact functional form of the scattering function in the frequency domain. Therefore, it is convenient to Fourier transform the spectra into the time domain. In this way, the resolution effect can easily be corrected by simple division of the Fourier transformed spectrum by that of the reference measurement corresponding to a purely elastic signal.

The deconvoluted intermediate scattering functions obtained from several spectra including those in Fig. 1(a) are shown in Fig. 1(b) of the figure. These functions decay in two steps. The first step relates to the above mentioned fast dynamical processes, which lead to a decay of the correlations at times of the order of the picosecond level. At this temperature, the methyl group dynamics is also expected to contribute at such short times. ${ }^{58}$ In the long-time regime $(t \geq 2 \mathrm{ps})$ the intermediate scattering function decays further, mainly dominated by the structural relaxation. It is wellknown by now that glass-forming dynamics associated to the structural relaxation does not show simple exponential behavior for the correlation functions but exhibits stretched spectra. As can be seen, after $\approx 1$ ps the scattering functions can be well described phenomenologically by a stretched exponential or Kohlrausch-Williams-Watts (KWW) functional form

$$
\phi_{\mathrm{KWw}}(t)=A \exp \left[-\left(\frac{t}{\tau_{w}(Q, T)}\right)^{\beta}\right] .
$$

Here $\beta$ is the stretching parameter accounting for the deviations from exponential behavior. It can take values between 0 and 1 and, for glass-forming polymers, is usually of the order of $0.5 .^{30} \tau_{w}$ is the characteristic time, which in general depends on both $Q$ and temperature. The prefactor $A$ is an effective Lamb-Mössbauer factor that parameterizes the contribution of the fast dynamics. The data in Fig. 1(b) have 


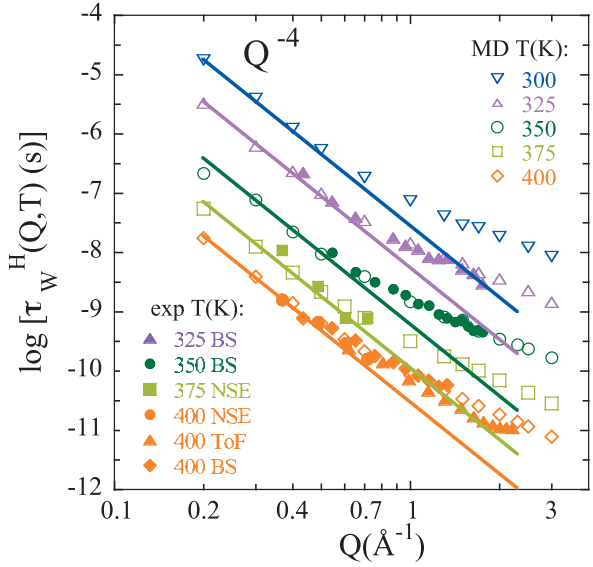

FIG. 2. $Q$-dependence of the characteristic relaxation times obtained from the KWW fit of the second decay of the intermediate scattering function at the temperatures indicated. Full symbols correspond to experimental data and empty symbols to simulation results. Solid lines represent a $Q^{-4}$-dependence. Simulated data are shifted by 0.5 decades toward faster times.

been described with a fixed value of the shape parameter $\beta$ equal to 0.5 (we have chosen this value because, as we will see below, it provides a good description of the validated simulations in a wide time range). The timescales $\tau_{w}(Q)$ resulting from these fits are displayed in Fig. 2 as full triangles (400 ToF). They show a strong variation with $Q$.

As can be appreciated in Fig. 1(b), the longest Fourier time accessible by FOCUS is of the order of 20 ps. With decreasing temperature, the dynamics of the $\alpha$-relaxation slows down and cannot be properly resolved by ToF techniques anymore. To characterize dynamical processes with characteristic timescales in the nanosecond range-e.g., at low temperatures and/or small $Q$-values-NSE or backscattering are more suitable since they offer a better resolution. Figure 3 shows as an example NSE results at several $Q$-values and the two temperatures investigated. ${ }^{59}$ The longer Fourier times accessed allow following well almost the complete decay of the scattering function in the experimental window and the univoque determination of the characteristic timescales. KWW fits [Eq. (8)] of the NSE data with $\beta$ $=0.5$ deliver the timescales shown in Fig. 2 as full circles $(400 \mathrm{~K})$ and full squares $(375 \mathrm{~K})$. Now considering the backscattering results, we note that their Fourier transformation is not straightforward, due to the rather limited dynamic window accessed by these instruments. Therefore, the IN16 data were directly fitted in the frequency domain with the Fourier transformed KWW functions $(\beta=0.5)$ convoluted with the instrumental resolution. Figure 4 shows the good quality of such fits for several $Q$ - and temperature values. The corresponding results for the timescales have been included in Fig. 2. We note that the agreement between the results obtained from different instruments at the common temperature is excellent, supporting the consistency of the analysis procedure followed.

The characteristic times in Fig. 2 follow the dispersion law $\tau_{w} \propto Q^{-4}$ in the $Q$-range below $\approx 0.7 \AA^{-1}$. As can be seen from inspection of Eq. (8), when a $\beta$-value of 0.5 is considered, this $Q$-dependence of $\tau_{w}$ implies the Gaussian func-

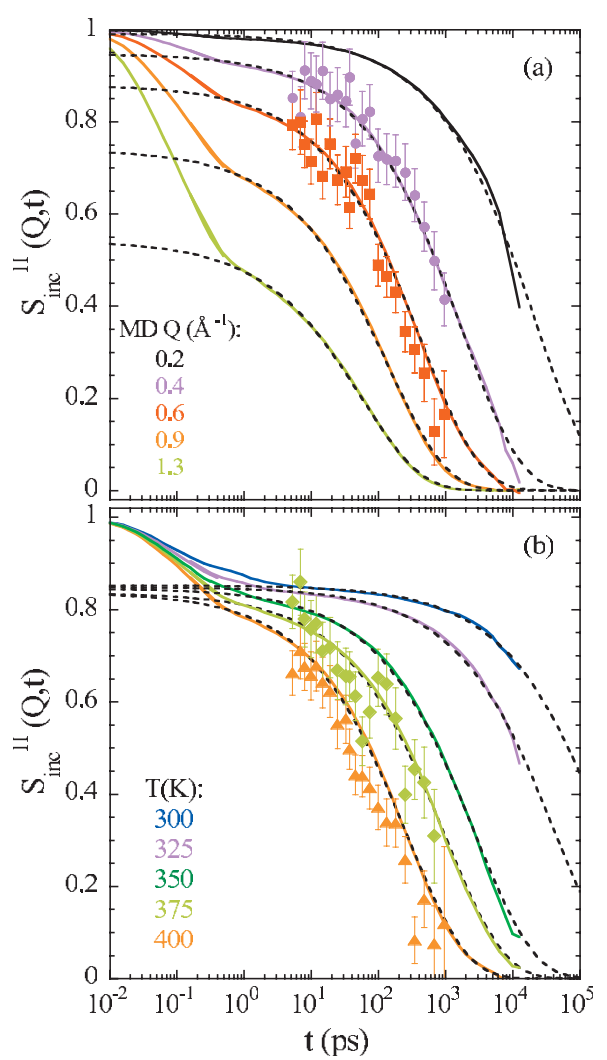

FIG. 3. Direct comparison between simulated (solid lines) and experimental (symbols) total hydrogen $S_{\text {inc }}^{\mathrm{H}}(Q, t)$. (a) NSE data at the values of $Q$ $=0.37 \AA^{-1}$ (circles) and $Q=0.6 \AA^{-1}$ (squares) are compared with simulated data at the indicated $Q$-values at $400 \mathrm{~K}$. (b) Simulated and experimental data are shown at $Q=0.72 \AA^{-1}$ at the different written temperatures. In both figures the simulated data are shifted by 0.5 decades toward faster times and the dashed lines represent the KWW fits.

tional form of the intermediate scattering function [Eq. (4)] and consequently of the self-part of the van Hove correlation function [Eq. (2)]. ${ }^{22}$ However, rather strong deviations are envisaged at larger $Q$-values. These can be better visualized with the construction of a master curve with the timescales corresponding to different temperatures by applying $T$-dependent shift factors. The result is shown in Fig. 5. We observe a crossover from a Gaussian $\left(\tau_{w} \propto Q^{-4}\right)$ to a weaker $Q$-dependence in the high- $Q$-regime. There, the scaled times can still be approximately described by a power law $\tau_{w} \approx Q^{-x}$, but the value found for the exponent $x$ is $x=2.5 \pm 0.5$.

\section{B. Simulations}

With the NS data at hand, the reliability of the simulated cell can easily be checked by calculating from the simulations the measured magnitudes and contrasting simulated and experimental results. Figure 3 shows the comparison between the intermediate scattering functions as measured by NSE and computed for all hydrogens in the simulation. To match both sets of data, the timescales of the simulations have been shifted by 0.5 decade (the experimental data decay at faster times). We can see that, allowing such a shift, the agreement is excellent. To extend this comparison to all $Q$-values and $T$-values investigated, we have analyzed the simulated data in the same way as the experimental results. 


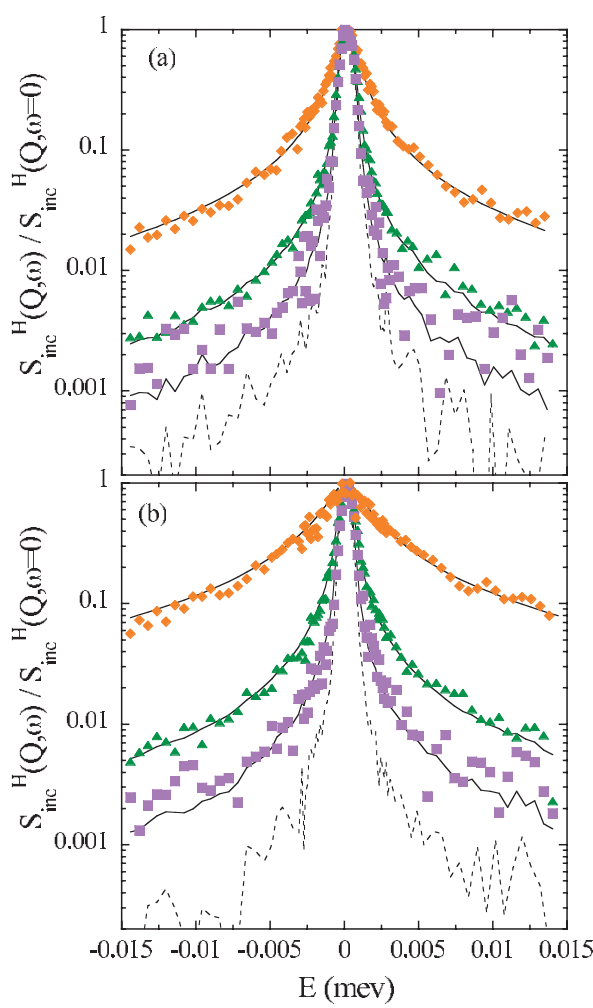

FIG. 4. Spectra obtained with IN16 measurements at the temperatures of 325 (squares), 350 (triangles) and $400 \mathrm{~K}$ (diamonds) at the different $Q$-values: (a) 0.652 and (b) $1.062 \AA^{-1}$. Spectra are normalized to their maximum intensities, solid lines are the fitting curves, and the dashed line is the instrumental resolution.

KWW functions with $\beta=0.5$ describe well the slow decay of the simulated intermediate scattering function (see, e.g., Fig. 3). The resulting timescales are compared to the experimental ones in Fig. 2. There, a shift of 0.5 decade toward faster times has been applied to all the MD-data. We can appreciate that, allowing this shift, the superposition with the experimental data is nearly perfect. Including the MD-results in the master curve of Fig. 5, we can also see how well the $Q$-dependence of the timescales is reproduced by the simulated cell.

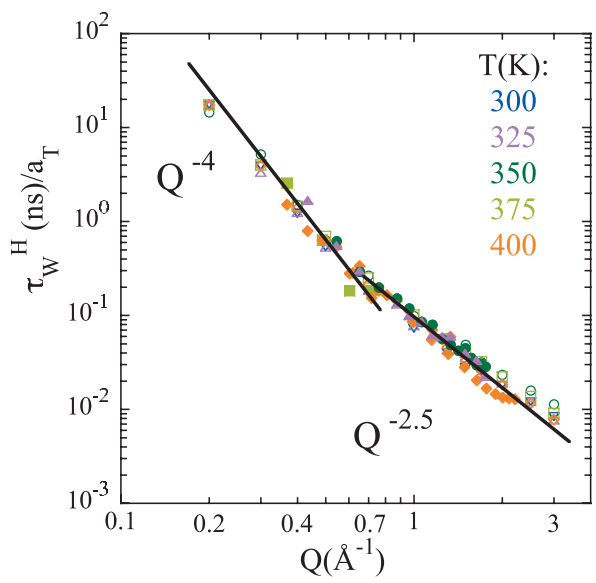

FIG. 5. Master curve constructed by shifting the experimental and simulated data of Fig. 2 by a shift factor $a_{T}$. Symbols as in Fig. 2. The lines show a $Q^{-4}$-law (Gaussian behavior) and a $Q^{-2.5}$-law approximately describing the high- $Q$ dependence.
We note that shifts in temperature and/or timescale are usually required for a perfect matching of results from MD-simulations and real samples. We can mention the cases of orthoterphenyl, ${ }^{60}$ polyisoprene, ${ }^{31}$ poly(methyl methacrylate), ${ }^{38,61}$ poly(vinyl acetate), ${ }^{34}$ and poly(ethylene-propylene). ${ }^{62}$ Apart from slight differences in the microstructure or in the density, a possible source for this discrepancy could be that the force field used in the simulations was not perfectly calibrated. For the case of PB, it has been shown by Smith et al. ${ }^{63}$ that the time scale of the $\alpha$-relaxation resulting from the MD-simulations is quite sensitive to slight changes in the local intrachain potentials. In this direction, the comparison with experiments as done here could be used to refine the rotational potentials for the force field for PVME.

Thus, from this comparison and the above discussion, we can state that, regarding the dynamics in the $\alpha$-relaxation regime, the simulated cell provides a good mimic of PVME behavior.

From the MD-trajectories it is possible to calculate also magnitudes that are not easily (or not at all) experimentally accessible, such as, e.g., correlation functions in real space, or address the self-motions of carbons. This information can be of utmost help to interpret experimental observations, or to check different theoretical frameworks. These capabilities of simulations are exploited in the next section.

\section{DISCUSSION}

Extending to lower $Q$-values, our experimental results support the finding of a Gaussian regime in the scattering function of PVME previously reported. ${ }^{21-23}$ Furthermore, they provide more clear indications of the deviations from Gaussian behavior at high- $Q$, which are well reproduced by the MD-simulations. Qualitatively similar $Q$-dependences have been reported for the characteristic times of a series of polymers, indicating a crossover from Gaussian to nonGaussian behavior in the range $0.6-1 \AA^{-1} \cdot{ }^{31-33,35,38,64,65}$ The information provided by MD-simulations has been of utmost help to shed some light on the origin of the deviations from the Gaussian approximation. ${ }^{31,33,35,37,38,56,64}$ For example, MD-simulations have evidenced the role played by the chemical heterogeneity inherent to real polymers in the emergence of such deviations [see, e.g., the cases of 1,2-PB (Ref. 35) and 1,4-PB (Ref. 36)]. In PVME, it is clear that methyl hydrogens will show additional motions with respect to the main-chain dynamics, ${ }^{58,66}$ and therefore their contribution to the scattering function will be a source for deviations from Gaussian behavior. ${ }^{67}$ Methyl group dynamics will be the subject of a separate study ${ }^{68}$ and here we will focus on the behavior of main-chain hydrogens $(\mathrm{cH})$. Simulations allow access to interesting functions to address this problem like the self-part of the van Hove correlation function $G_{\text {self }}^{\mathrm{cH}}(r, t)$ [Eq. (1)] and its moments [Eq. (3)]. Figure 6 shows the evolution with time of $G_{\text {self }}^{\mathrm{cH}}(r, t)$ at the lowest and highest temperatures investigated. At $300 \mathrm{~K}$, a strong localization of the hydrogen motions in a region within $\approx 2 \AA$ from its initial position persists for several nanoseconds. Close to the limit of the simulations, at $20 \mathrm{~ns}$, although the main peak is 


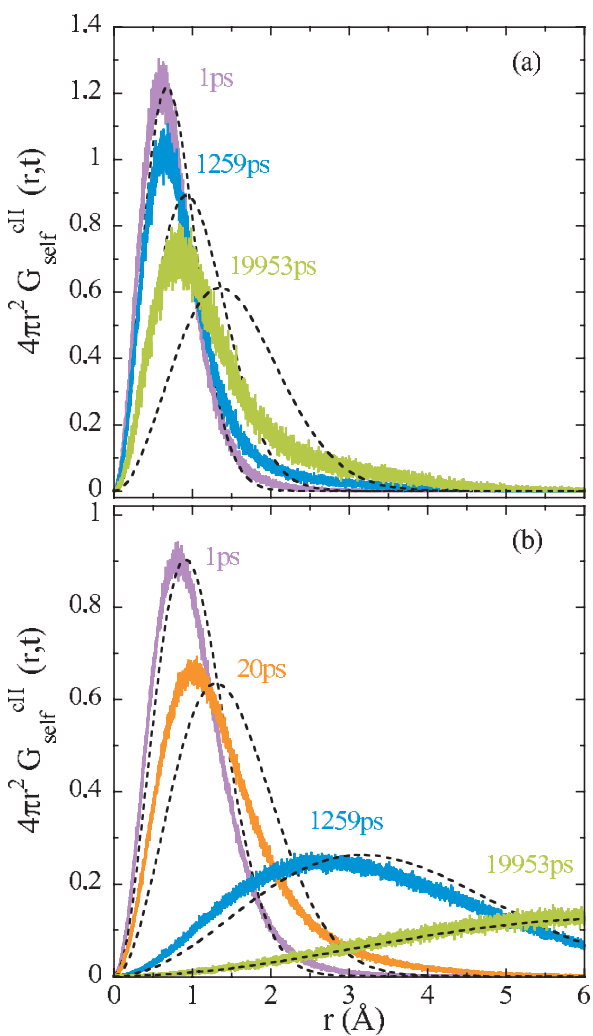

FIG. 6. Self-part of the $\mathrm{cH}$ van Hove correlation function for the different times indicated at the temperatures of (a) 300 and (b) 400. Dashed lines represent the Gaussian approximation, in which the mean squared displacements are the simulated ones at the corresponding time.

still centered below $1 \AA$, the distribution function becomes slightly broader and shifted toward longer distances. This can be regarded as a signature of the onset of a diffusivelike process. At $400 \mathrm{~K}$ the behavior is rather different. With increasing time, the function continuously broadens and shifts toward larger values of the distance, revealing a predominantly diffusive character of the underlying dynamics. This qualitatively different behavior at both extreme temperatures is reflected in the development of the mean squared displacement $\left\langle r_{\mathrm{cH}}^{2}(t)\right\rangle$ [Eq. (3), see Fig. 7]. For the $300 \mathrm{~K}$ data we see that, after the rapid increase in $\left\langle r_{\mathrm{cH}}^{2}(t)\right\rangle$ in the fast-dynamics regime (below $\approx 1 \mathrm{ps}$ ), it stays almost constant for about

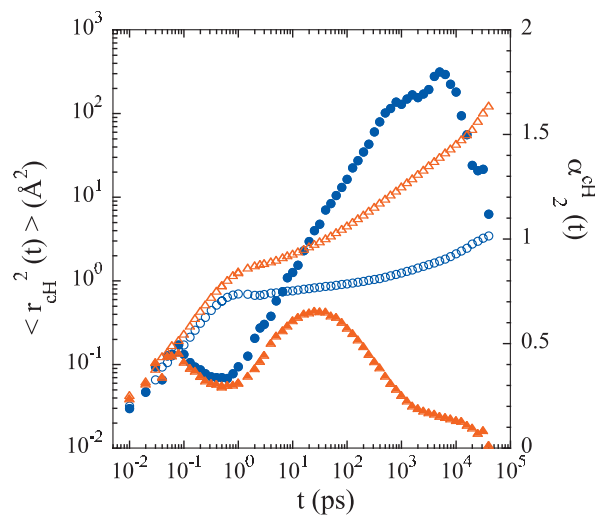

FIG. 7. Time evolution of the mean squared displacements (empty symbols) and of the non-Gaussian parameter $\alpha_{2}(t)$ (full symbols) at 300 (circles) and $400 \mathrm{~K}$ (triangles) of the cHs.

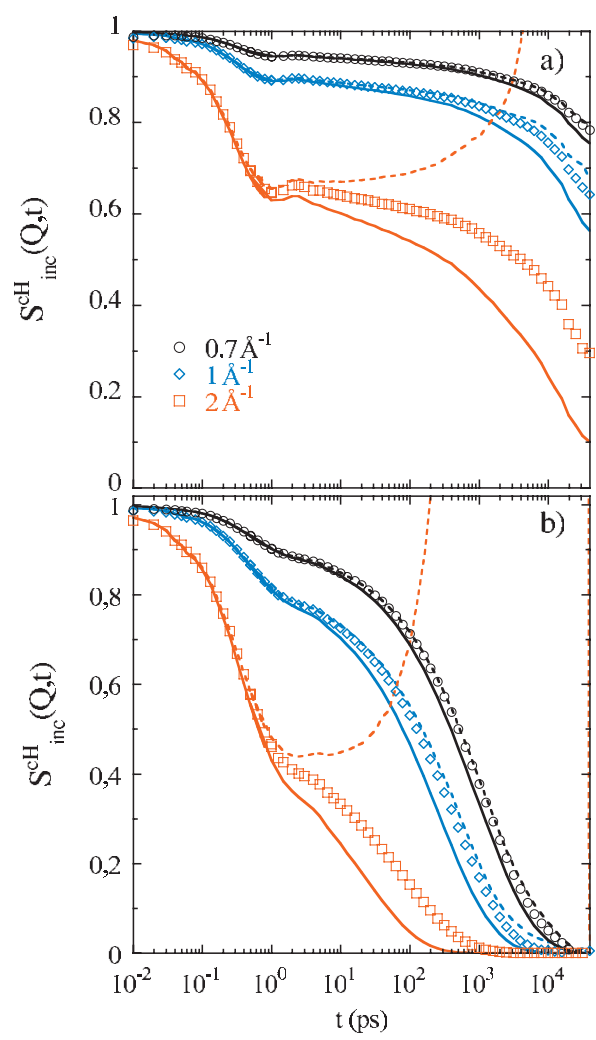

FIG. 8. cH self-correlation function at (a) 300 and (b) $400 \mathrm{~K}$. Symbols represent the scattering function $S_{\text {inc }}^{\mathrm{cH}}(Q, t)$ at the different $Q$-values indicated, solid lines represent the Gaussian approximation, and dashed lines include the first correction of the $Q^{2}$-expansion of the scattering function.

three decades in time, and only after $\approx 3$ ns does it start increasing significantly. In the intermediate times region between $\approx 1 \mathrm{ps}$ and $3 \mathrm{~ns}$, the hydrogens are thus trapped within the cage imposed by the neighbors. The duration of this regime is strongly reduced at high temperatures. At $400 \mathrm{~K}$, the mean squared displacement shows a well-developed sublinear regime characterized by $\left\langle r_{\mathrm{cH}}^{2}(t)\right\rangle \propto t^{0.5}$ that begins already at around $20 \mathrm{ps}$ (see Fig. 7). This sublinear regime is characteristic for glass-forming polymer dynamics (see, e.g., Ref. 56) and is attributed to the segmental dynamics crossing over Rouse motion at longer times. ${ }^{69}$

We now can check the validity of the Gaussian approximation in real space [Eq. (2)]. In Fig. 6 the calculated $G_{\text {self }}^{\mathrm{cH}}(r, t)$ are directly compared with the Gaussian functions [Eq. (2)] displaying the corresponding value of the mean squared displacement (represented in Fig. 7). At short times the agreement could be acceptable, as well as for the longest times at $400 \mathrm{~K}$. At this temperature, the deviations are largest in the region of $10-100$ ps. For $300 \mathrm{~K}$ we find severe deviations from the Gaussian approximation for all times accessed above some picoseconds.

In an analogous way, the Gaussian approximation can be checked in the reciprocal space, comparing the intermediate scattering function with Eq. (4). The results are shown for three $Q$-values at $300 \mathrm{~K}$ in Fig. $8(\mathrm{a})$ and at $400 \mathrm{~K}$ in Fig. 8(b). It is clear that for high $Q$-values (around $2 \AA^{-1}$ ) the scattering functions strongly deviate from the Gaussian approximation already from rather short times (picoseconds). The deviations almost vanish at $Q \approx 0.7 \AA^{-1}$ but are still 
noticeable around $1 \AA^{-1}$. We may consider the leading correction in the expansion [Eq. (5)] with the values of the $\alpha_{2}(t)$ parameter [Eq. (6)] calculated from the moments of $G_{\text {self }}^{\mathrm{cH}}(r, t)$ (Fig. 7). As can be seen in Fig. 8, the $\alpha_{2}$-correction [Eq. (5)] is enough to account for the deviations of $S_{\text {inc }}^{\mathrm{cH}}(Q, t)$ at $Q$ $\approx 0.7 \AA^{-1}$. However, this first correction is clearly insufficient at higher $Q$-values, and further terms would be needed to reproduce the behavior of $S_{\text {inc }}^{\mathrm{cH}}(Q, t)$, even at $Q \approx 1 \AA^{-1}$.

We note that also in absence of methyl group rotations, as it is the case of the $\mathrm{cH}$, the incoherent scattering function displays a two-step decay. This is one of the main predictions of MCT. In addition, in the previous discussion of the correlation functions in real space we have found strong evidences for the cage effect-another main ingredient of this theory. These observations suggest trying to check MCT predictions with our simulation data - if applicable, it should also give account for the observed deviations from Gaussian behavior of the intermediate scattering function.

\section{A. MCT analysis}

In principle we cannot expect that PVME dynamics can be described by the original MCT of Bengtzelius et al., ${ }^{70}$ which was formulated for a hard-sphere-like liquid. However, a phenomenological MCT version, solely relying on the theorems derived in Ref. 2, might be applied. Here we will just invoke some of the main predictions of this MCT version that will be tested with our data. As a correlation function $\phi_{Q}(t)$, we will consider the self-correlation function of three different subsystems of atoms: $\mathrm{cH}$, main-chain carbons $(\mathrm{cC})$, and all the hydrogens $(\mathrm{H})$.

A prediction that can be tested without invoking fits or knowledge of the values of the critical parameters is the factorization in the $\beta$-regime: $\phi_{Q}(t)=f_{Q}+h_{Q} G(t)$. If it holds, the ratio

$$
R_{Q}(t)=\frac{\phi_{Q}(t)-\phi_{Q}\left(t^{\prime}\right)}{\phi_{Q}\left(t^{\prime \prime}\right)-\phi_{Q}\left(t^{\prime}\right)}
$$

must be $Q$-independent. Figure 9 shows that for $300 \mathrm{~K}$ the superposition of these functions is almost perfect in the region 5 ps $\lesssim t \lesssim 3$ ns (MCT- $\beta$ regime). Here we have considered all hydrogens [Fig. 9(a)] and also the $\mathrm{cH}$ [Fig. 9(b)]. For $\mathrm{cC}$, the same quality of superposition is achieved.

The factorization theorem can also be checked in real space, using the van Hove correlation functions. It reads $G(r, t)=F(r)+H(r) G(t)$. In the $\beta$-regime, the ratio

$$
R(r, t)=\frac{G(r, t)-G\left(r, t^{\prime}\right)}{G\left(r^{\prime}, t\right)-G\left(r^{\prime}, t^{\prime}\right)}=\frac{H(r)}{H\left(r^{\prime}\right)}
$$

should be independent of time ${ }^{71}$ and inform us about the length scales involved in this process (for the self-atomic motions in our case). In Fig. 10 we can see the good master curves obtained considering times in the interval between 10 and $400 \mathrm{ps}$. At faster and longer times (about $700 \mathrm{ps}$ ) failure of the superposition is observed. Thus, master curves in real space are only obtained in the intermediate time window of the plateau (see Fig. 9). A similar situation was reported for a model polymer melt ${ }^{72}$ and the binary Lennard-Jones mixture. ${ }^{73}$ Figure 10 (a) shows that the motions of cCs are

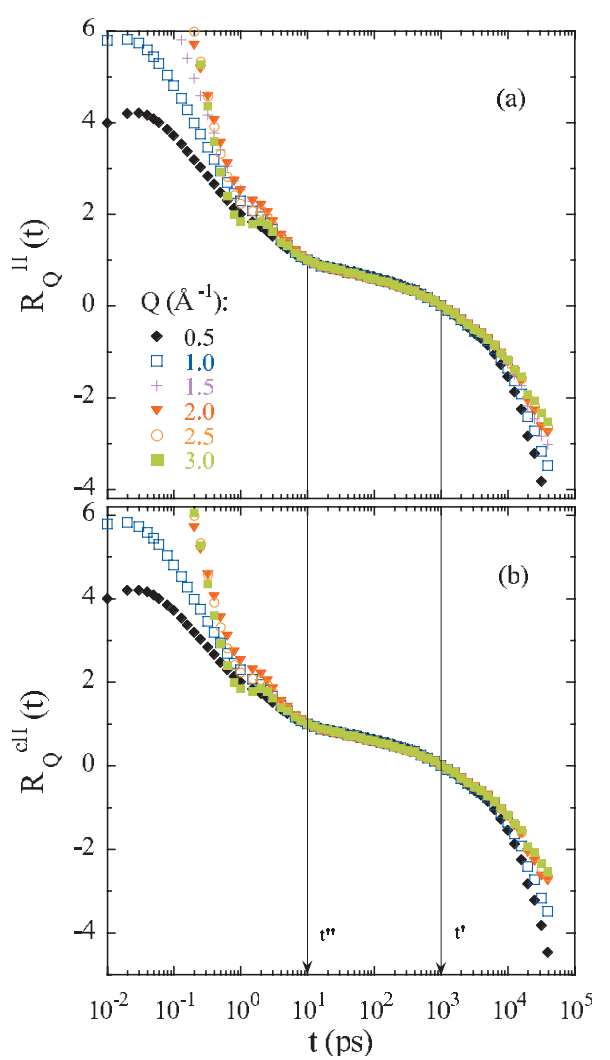

FIG. 9. Factorization theorem applied to the correlation function $\phi_{Q}(t)$ of (a) all hydrogens and (b) of the $\mathrm{cHs}$ at $300 \mathrm{~K}$ at the indicated $Q$-values. $t^{\prime}$ and $t^{\prime \prime}$ represent the limits of the $\beta$-window

limited to displacements smaller than $3 \AA$. This value is clearly smaller than the average intermolecular distance in this polymer, which can be estimated in the Bragg approximation from the first peak position observed in the simulated structure factor $\left(Q_{1 \max }=1 \AA^{-1}\right)$. We obtain $d_{\text {chain }}$ $\approx 2 \pi / Q_{1 \max }=6.3 \AA$. At small distances, the function $R^{c C}(r, t)$ shows positive values with a peak, vanishes at $r_{o}$ $=0.76 \AA$, and displays a negative peak at longer distances. We note that the function $\mathrm{H}(r)$ determines the rate at which the van Hove correlation relaxes ${ }^{14}$

$$
\frac{\partial G(r, t)}{\partial t} \propto-\mathrm{H}(r)
$$

This implies that in the $\beta$-regime the probability for atomic displacements of size $r$ decreases most where $\mathrm{H}(r)$ is largest $(r \approx 0.4 \AA)$, and increases most where $\mathrm{H}(r)$ is smallest $(r \approx 1.1 \AA)$. This last distance is much smaller than $d_{\text {chain }}$; therefore, attempts to overcome distances of the order of the intermolecular separation are partially reflected back and the atom returns close to its initial position. ${ }^{14,74}$

For $\mathrm{cH}$, the behavior is qualitatively identical to that shown by $\mathrm{cC}$, though the delocalization of hydrogens is slightly larger [Fig. 10(b)]. If we consider all hydrogens [Fig. 10(c)], a second negative peak appears at a longer distance. This corresponds to the other two equivalent positions of methyl group hydrogens, which are also visited during the time interval considered. The methyl group rotates within the cage imposed by the neighboring chains. 

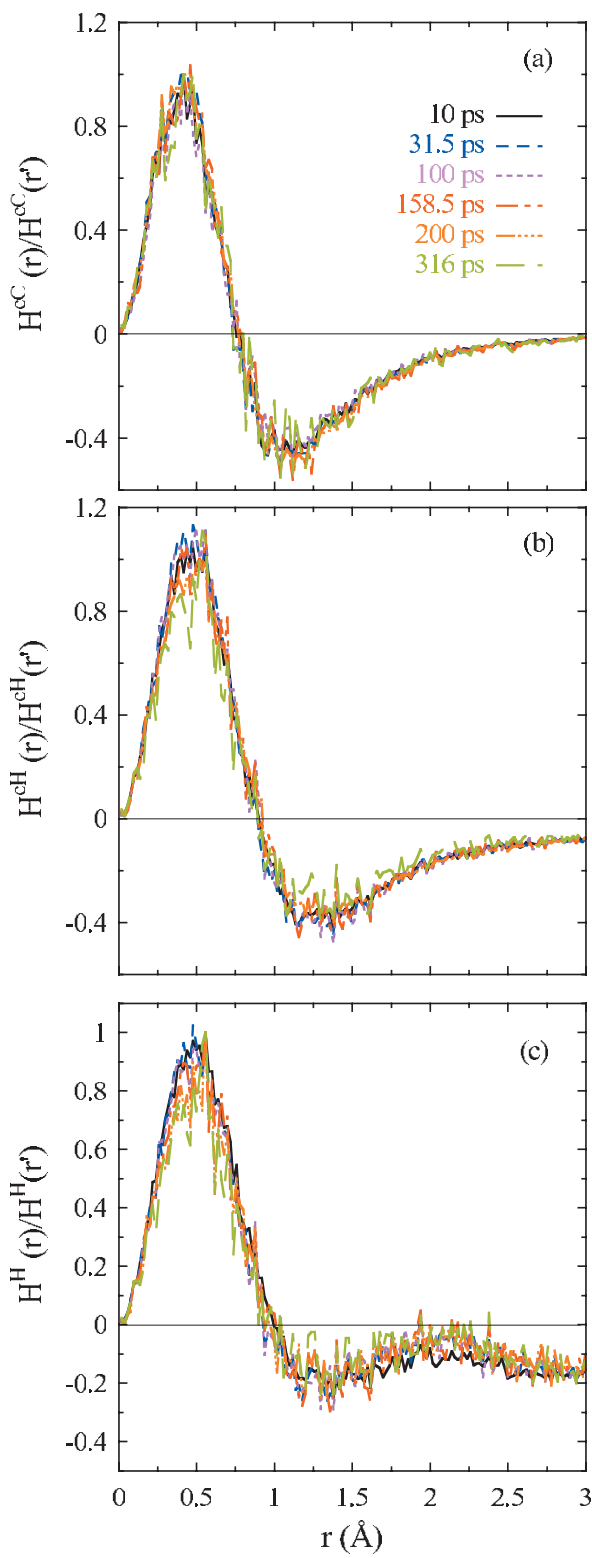

FIG. 10. Factorization theorem in the real space for the self-part of the van Hove scattering function evaluated for (a) the cCs, (b) cHs, and (c) for all hydrogens. The theorem has been applied to simulated data at $300 \mathrm{~K}$ at the different indicated times. The values of $r^{\prime}\left[r^{\prime}(c C)=0.42 \AA, \quad r^{\prime}(\mathrm{cH})\right.$ $=0.52 \AA$, and $\left.r^{\prime}(\mathrm{H})=0.56 \AA\right]$ have been chosen such that the denominator in Eq. (10) is maximized.

Another expectation of MCT is that in the $\alpha$-regime, the correlation functions obey the time/temperature superposition principle. In Fig. 11 this property is checked for the hydrogen self-motions at the first peak structure factor $Q$-value. The timescale chosen to superimpose the data is that where the correlation function takes the value $0.2 .^{75}$ The data corresponding to $300 \mathrm{~K}$ have not been included because at this $Q$-value, the decay only reaches the value of 0.5 in the limit of the simulated window, and the characteristic time cannot be properly determined to scale the function. For the temperatures considered, we observe a rather good superposition in the long-time region.

Now we obtain the values of the critical parameters for PVME and test the consistency of other MCT predictions by studying the behavior of parameters that can easily be ob-

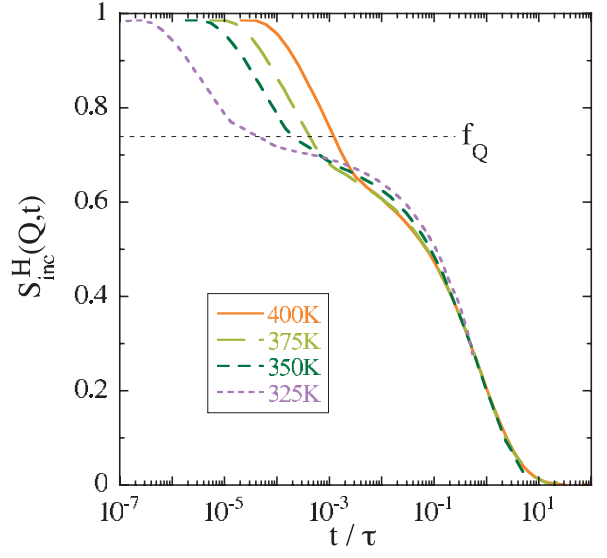

FIG. 11. Check of the time-temperature superposition principle for $\phi_{Q}(t)$ at the first peak structure factor $Q$-value $\left(Q_{\max }=1 \AA^{-1}\right)$ at the indicated temperatures.

tained from a phenomenological data analysis. From the fit of the last stage of the decay of the correlation functions by a KWW function [Eq. (8)] the $Q$-dependent and $T$-dependent shape parameter $\beta$ can be obtained. The resulting values for two temperatures are shown in Fig. 12. The value of the von Schweidler exponent $b$ in the MCT can be deduced as the high- $Q$ limit of this parameter, ${ }^{76}$

$$
\lim _{Q \rightarrow \infty} \beta(Q)=b .
$$

As the KWW exponent of the three correlators is in the region between 0.3 and 0.4 for high $Q$, we have chosen 0.35 as a good compromise for the $b$ exponent. From the MCT relations

$$
\lambda=\frac{\Gamma^{2}(1+b)}{\Gamma(1+2 b)}=\frac{\Gamma^{2}(1-a)}{\Gamma(1-2 a)}, \quad \gamma=\frac{1}{2 a}+\frac{1}{2 b},
$$

values of $\lambda=0.87$ and $\gamma=3.6$ are obtained. They are relatively large as compared to those reported for other systems; this point will be discussed below.

The $\gamma$-parameter determines the way the characteristic time for the structural relaxation approaches the critical temperature $T_{c}$

$$
\tau \propto\left|T-T_{c}\right|^{-\gamma} .
$$

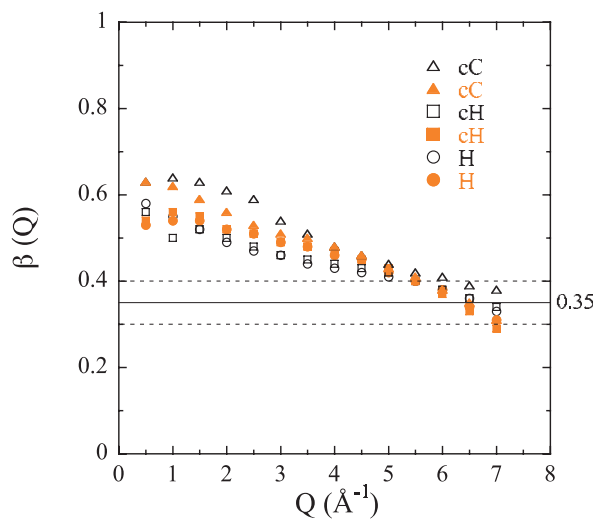

FIG. 12. $Q$-dependence of the stretching parameter $\beta$ evaluated at 325 (empty symbols) and $350 \mathrm{~K}$ (full symbols) of $\phi_{Q}(t)$ of the cCs (triangles), cHs (squares), and all hydrogens (circles). 


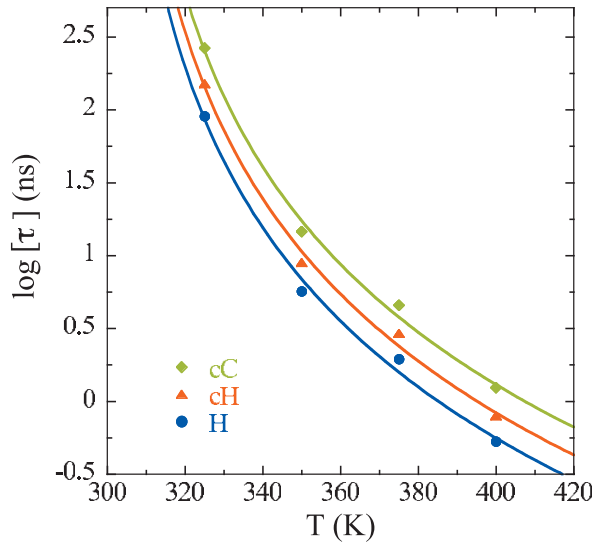

FIG. 13. Temperature dependence of the characteristic times in which the different $\phi_{Q}(t)$ correlation functions reach the value of 0.2 . The solid lines show the fit according to Eq. (14).

A priori, we do not know the value of $T_{c}$ for our system. In the literature is usually found that $T_{c} \approx 1.2 T_{g}$. Considering the glass-transition temperature of the real sample, this would correspond to $T_{c} \approx 300 \mathrm{~K}$. We have determined the value of $T_{c}$ in our system by applying Eq. (14) to the timescales defined as those where the correlation functions take the value of 0.2 (Fig. 13). As previously mentioned, at $300 \mathrm{~K}$ this timescale cannot be properly determined and therefore, it has not been considered. Moreover, according to the rough estimation of $T_{c}, 300 \mathrm{~K}$ should be very close to the value of the MCT- $T_{c}$. As can be seen in Fig. 13, the value of $\gamma$ describes very well the $T$-dependence of the times. The fits deliver a value of $T_{c} \approx 300 \mathrm{~K}$. Thus, from this first analysis we can see that the PVME results are compatible with MCT predictions assuming a rather large value of the exponent parameter $\lambda$ and $T_{c}=300 \mathrm{~K}$, as would be expected from the experimental $T_{g}$-value.

In the following, we will thus assume that the MCT can be applied to our data with the critical exponents determined from the previous analysis. Being the $\beta$-scaling fulfilled, a description of the decay from the plateau by means of the MCT von Schweidler expansion

$$
\phi_{Q}(t)=f_{Q}-\mathrm{H}_{1 Q} t^{b}+\mathrm{H}_{2 Q} t^{2 b}+\cdots
$$

should be possible with a $T$-independent critical nonergodicity parameter $f_{Q}$. We have first considered the correlation functions at different temperatures. For each $Q$-value, we have described all the curves with a single value of $f_{Q}$ (see Fig. 14 for the case of $\mathrm{cC}$ ). The resulting $f_{Q^{-}}$-values for the different subsystems considered are displayed in Fig. 15. Those corresponding to main-chain atoms follow well a Gaussian behavior. Assuming $f_{Q}=\exp \left(-Q^{2} r_{s c}^{2}\right)$, localization lengths of $r_{s c}=0.28$ and $0.47 \AA$ are deduced for carbons and hydrogens, respectively. The deviations from Gaussian dependence observed for $f_{Q}$ when all hydrogens are considered stem from the methyl group rotational contributions. The fact that the incoherent scattering function can be reasonably described by Eq. (15) with $T$-independent $f_{Q}$-values supports again the applicability of MCT predictions to this polymer data.

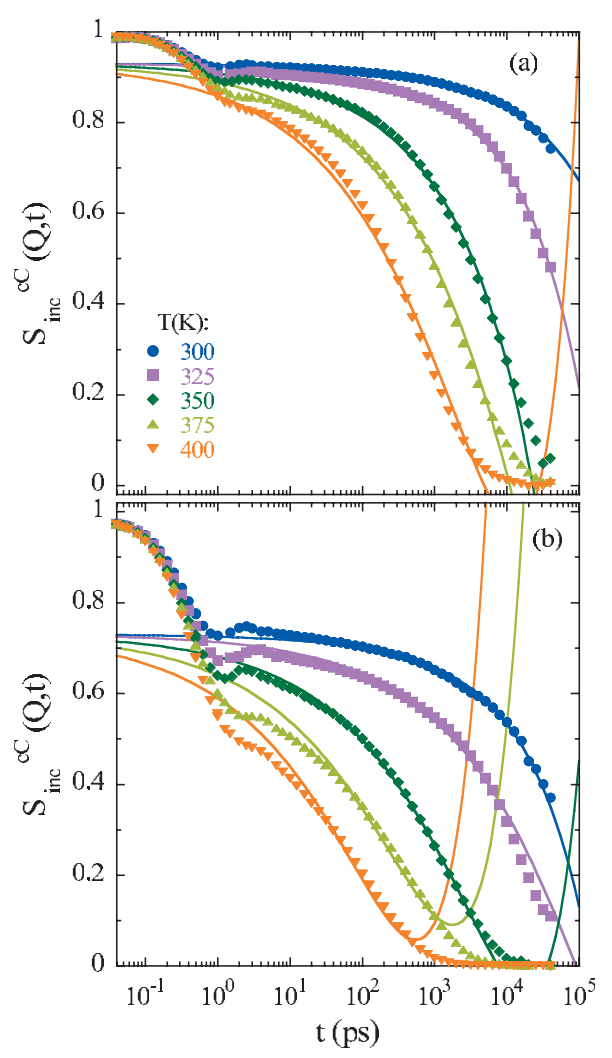

FIG. 14. Fit of the MCT von Schweidler regime of the $S_{i n c}^{c C}(Q, t)$ of the cCs at (a) $Q=1.0 \AA^{-1}$ and (b) $Q=2.0 \AA^{-1}$ at the written temperatures. All the curves are described with a single value of the nonergodicity parameter $f_{Q}$.

We can further check whether the $H_{1 Q}$ parameters in Eq. (15) fulfill the relation

$$
H_{1 Q} \propto\left|T-T_{c}\right|^{\gamma b} .
$$

Figure 16 shows the good agreement between the obtained

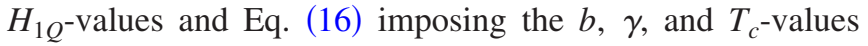
previously deduced. We remind that these were obtained from the shape parameter at high- $Q$ and the $T$-dependence of the $\alpha$-relaxation timescale, i.e., in an independent way. Finally, we note that, consistently, the observed high- $Q$ limit for the characteristic timescales obtained from both, simula-

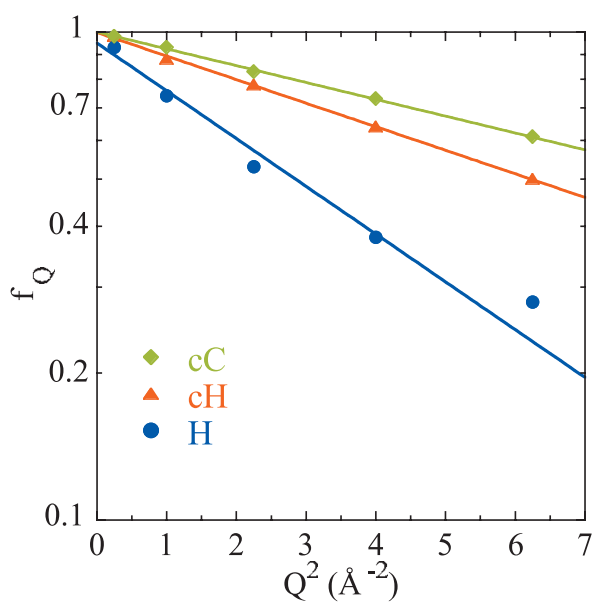

FIG. 15. $Q$-dependence of the nonergodicity parameter $f_{Q}$ calculated for the different atomic species. Solid lines represent the fit with the expression $f_{Q}=\exp \left(-Q^{2} r_{s c}^{2}\right)$. 


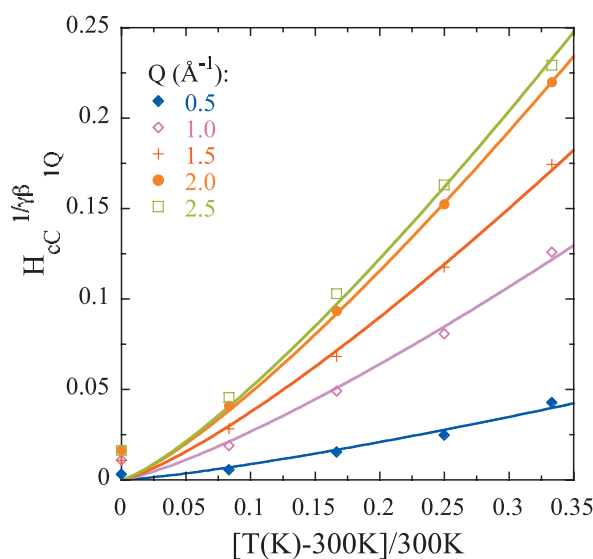

FIG. 16. Temperature dependence of the $H_{1 Q}$-parameter of the MCT von Schweidler expansion of $\phi_{Q}(t)$ for cCs at different $Q$-values. The solid lines show the fit with Eq. (16).

tions and experiments (Fig. 5), would be well compatible with the $Q^{-1 / b}=Q^{-2.86}$ also predicted by this theory. ${ }^{77}$ Thus, the deviations from Gaussian behavior found in experiments and MD-simulations could be understood within the general framework provided by the MCT.

We have thus obtained very consistent results in this phenomenological approach to the application of MCT. The value here deduced for the exponent parameter $\lambda=0.87$ is high as compared to other glass-forming systems. ${ }^{11,60,78,79}$ Low-molecular weight systems, as $o$-terphenyl, silica, or water, show values similar to that of hard spheres $(\lambda=0.74)$. We note that in a similar way as for PVME, a rather high $\lambda$-value (0.93) has recently been reported for fully atomistic 1,4-PB. ${ }^{18}$ In that work, such finding was attributed to the local conformational changes controlled by rotational barriers, which affect the dynamics of the polymer segments within the cage. Similar results are found for $1,2-\mathrm{PB} .^{80}$ It is worth noting that chain connectivity in bead-spring models does not yield significant changes in $\lambda$. In fact, in a recent work on MD-simulations of a simple model for polymer melts with intramolecular barriers ${ }^{20}$ it has been found that there is a clear correlation between the exponent parameter and chain stiffness. In their interpretation, the competition between packing effects and intramolecular barriers is the mechanism responsible for the increase in $\lambda$ from 0.76 for fully flexible chains (like the bead-spring models referred to in Ref. 14 that do not include rotational barriers) to $\lambda \approx 0.90$ for very stiff chains. Chemically realistic polymer models seem to exhibit $\lambda$-values approaching the limit $\lambda=1$. For example, a united atom model of polyethylene ${ }^{16}$ reveals $\lambda=0.81$; for $1,4-\mathrm{PB}$, values of 0.90 and 0.93 have been reported from a united atom model as well, ${ }^{17}$ and a fully atomistic model, ${ }^{18}$ respectively. To check the importance of the torsional barriers in our simulated system, we had them "switched off" and analyzed the obtained results again in the light of the MCT. The value of the $\lambda$-parameter has decreased to $\lambda \approx 0.8$, showing thus the tendency suggested in work. ${ }^{20}$ We can then conclude that this study on PVME strongly supports the scenario proposed in Ref. 20, where real polymers would be classified in the family of complex systems as short-ranged attractive colloids ${ }^{81-83}$ or binary mixtures with strong dynamic asymmetry. ${ }^{84-87}$ The unusually large $\lambda$-values exhibited by them could reveal an underlying high-order MCT transition.

Finally, we note that the deviations from Gaussian behavior shown by the characteristic timescales of several polymers at high- $Q$ could be described by asymptotic powerlaws $Q^{-x}$ with $x \approx 2, \ldots, 2.5$. We can cite the cases of polyisobutylene, ${ }^{32}$ polyisoprene, ${ }^{31,56} 1,2-\mathrm{PB},{ }^{35}$ poly(methyl methacrylate), ${ }^{37,38}$ poly(ethylene-propylene), ${ }^{65}$ head-to-head polypropylene, ${ }^{65}$ and poly(vinyl acetate) ${ }^{64}$ As the intermediate scattering functions are usually described with $\beta$-values close to 0.5 , assuming that the $b$-parameter is not very different from $\beta$, this kind of behavior would be compatible with the $Q^{-1 / b}$-dependence predicted by MCT also in other polymer systems.

\section{CONCLUSIONS}

We have presented NS results from three different spectrometers on a protonated PVME sample, that have allowed characterizing its hydrogen dynamics above the glasstransition temperature. Deviations from Gaussian behavior have been found in the high- $Q$ range. Properly validated fully atomistic MD-simulations have allowed further characterization of these deviations accessing the correlation functions directly in real space.

Furthermore, the applicability of MCT to PVME data has been explored. We have obtained consistent results in the application of MCT phenomenological predictions to the self-correlation functions of PVME atoms calculated from our simulation data. The unusually large $\lambda$-value found is close to that reported for 1,4-PB (Ref. 18) and simple polymer models with intramolecular barriers. ${ }^{20}$ Such finding could be interpreted in the framework of a higher-order MCT transition for real polymers arising from the simultaneous occurrence of two mechanisms leading to dynamic arrest: (i) packing of intermolecular character and present in all glassforming systems; (ii) barriers for conformational changes of intramolecular origin, which are specific of macromolecular systems. ${ }^{18,20}$ Analogous analysis in other realistic polymer models or even with experimental data would allow confirming the universality of this framework.

\section{ACKNOWLEDGMENTS}

We thank the Institut Laue-Langevin (ILL) for neutron facilities, as well as Dr. P. Fouquet and Dr. M. Maccarini for experimental support at IN11C. S.C. acknowledges the grant of the Donostia International Physics Center (DIPC). We thank support by the DIPC, the European Commission NoE SoftComp Contract No. NMP3-CT-2004-502235, the Project Nos. MAT2007-63681 and IT-436-07 (GV), and the Spanish Ministerio de Educacion y Ciencia (Grant No. CSD2006-53).

\footnotetext{
${ }^{1}$ W. Götze and L. Sjögren, Transp. Theory Stat. Phys. 24, 801 (1995).

${ }^{2}$ W. Götze, in Liquids, Freezing, Glass Transition, edited by J. P. Hansen, D. Levesque, and J. Zinn-Justin (North-Holland, Amsterdam, 1991).

${ }^{3}$ W. Götze, J. Phys.: Condens. Matter 11, A1 (1999).

${ }^{4}$ W. Götze and L. Sjögren, Phys. Rev. A 43, 5442 (1991).

${ }^{5}$ G. Foffi, W. Götze, F. Sciortino, P. Tartaglia, and T. Voigtmann, Phys. Rev. E 69, 011505 (2004).

${ }^{6}$ J.-L. Barrat and A. Latz, J. Phys.: Condens. Matter 2, 4289 (1990).
} 
${ }^{7}$ M. Nauroth and W. Kob, Phys. Rev. E 55, 657 (1997).

${ }^{8}$ S. H. Chong, W. Götze, and A. P. Singh, Phys. Rev. E 63, 011206 (2000)

${ }^{9}$ R. Schilling and T. Scheidsteger, Phys. Rev. E 56, 2932 (1997).

${ }^{10}$ L. Fabbian, A. Latz, R. Schilling, F. Sciortino, P. Tartaglia, and C. Theis, Phys. Rev. E 62, 2388 (2000).

${ }^{11}$ T. Franosch, M. Fuchs, W. Götze, M. R. Mayr, and A. P. Singh, Phys. Rev. E 56, 5659 (1997).

${ }^{12}$ A. Tölle, H. Schober, J. Wuttke, and F. Fujara, Phys. Rev. E 56, 809 (1997)

${ }^{13}$ D. Richter, B. Frick, and B. Farago, Phys. Rev. Lett. 61, 2465 (1988).

${ }^{14}$ J. Baschnagel and F. Varnik, J. Phys.: Condens. Matter 17, R851 (2005).

${ }^{15}$ S.-H. Chong and M. Fuchs, Phys. Rev. Lett. 88, 185702 (2002).

${ }^{16}$ A. Van Zon and S. W. De Leeuw, Phys. Rev. E 60, 6942 (1999).

${ }^{17}$ W. Paul, D. Bedrov, and G. D. Smith, Phys. Rev. E 74, 021501 (2006)

${ }^{18} \mathrm{~J}$. Colmenero, A. Narros, F. Alvarez, A. Arbe, and A. J. Moreno, J. Phys.: Condens. Matter 19, 205127 (2007).

${ }^{19}$ W. Paul and G. D. Smith, Rep. Prog. Phys. 67, 1117 (2004).

${ }^{20}$ M. Bernabei, A. J. Moreno, and J. Colmenero, Phys. Rev. Lett. 101, 255701 (2008).

${ }^{21}$ J. Colmenero, A. Alegría, J. M. Alberdi, F. Alvarez, and B. Frick, Phys. Rev. B 44, 7321 (1991).

${ }^{22}$ J. Colmenero, A. Alegría, A. Arbe, and B. Frick, Phys. Rev. Lett. 69, 478 (1992)

${ }^{23}$ J. Colmenero, A. Arbe, A. Alegría, and K. L. Ngai, J. Non-Cryst. Solids 172-174, 229 (1994).

${ }^{24}$ A. Arbe, J. Colmenero, M. Monkenbusch, and D. Richter, Phys. Rev. Lett. 81, 590 (1998).

${ }^{25}$ H. Sillescu, J. Non-Cryst. Solids 243, 81 (1999).

${ }^{26}$ M. D. Ediger, Annu. Rev. Phys. Chem. 51, 99 (2000)

${ }^{27}$ S. C. Glotzer, J. Non-Cryst. Solids 274, 342 (2000).

${ }^{28}$ J. Colmenero, A. Arbe, A. Alegría, M. Monkenbusch, and D. Richter, J. Phys.: Condens. Matter 11, A363 (1999).

${ }^{29}$ J. Colmenero, A. Arbe, F. Alvarez, M. Monkenbusch, D. Richter, B. Farago, and B. Frick, J. Phys.: Condens. Matter 15, S1127 (2003).

${ }^{30}$ D. Richter, M. Monkenbusch, A. Arbe, and J. Colmenero, Neutron Spin Echo in Polymer Systems, Advances in Polymer Science (SpringerVerlag, Berlin, 2005), Vol. 174

${ }^{31}$ A. Arbe, J. Colmenero, F. Alvarez, M. Monkenbusch, D. Richter, B Farago, and B. Frick, Phys. Rev. E 67, 051802 (2003).

${ }^{32}$ B. Farago, A. Arbe, J. Colmenero, R. Faust, U. Buchenau, and D. Richter, Phys. Rev. E 65, 051803 (2002).

${ }^{33}$ A. Arbe, J. Colmenero, F. Alvarez, M. Monkenbusch, D. Richter, B. Farago, and B. Frick, Phys. Rev. Lett. 89, 245701 (2002).

${ }^{34}$ M. Tyagi, A. Arbe, F. Alvarez, J. Colmenero, and M. A. Gonzalez, J. Chem. Phys. 129, 224903 (2008).

${ }^{35}$ A. Narros, F. Alvarez, A. Arbe, J. Colmenero, D. Richter, and B. Farago, J. Chem. Phys. 121, 3282 (2004).

${ }^{36}$ A. Narros, A. Arbe, F. Alvarez, J. Colmenero, and D. Richter, J. Chem. Phys. 128, 224905 (2008).

${ }^{37}$ A.-C. Genix, A. Arbe, F. Alvarez, J. Colmenero, L. Willner, and D. Richter, Phys. Rev. E 72, 031808 (2005).

${ }^{38}$ A.-C. Genix, A. Arbe, F. Alvarez, J. Colmenero, B. Farago, A. Wischnewski, and D. Richter, Macromolecules 39, 6260 (2006).

${ }^{39}$ R. Casalini and C. M. Roland, J. Chem. Phys. 119, 4052 (2003).

${ }^{40}$ T. Springer, Quasielastic Neutron Scattering for the Investigation of Diffusive Motions in Solids, Liquids, Springer Tracts in Modern Physics (Springer-Verlag, Berlin, 1972), Vol. 64.

${ }^{41}$ S. W. Lovesey, Theory of Neutron Scattering from Condensed Matter (Clarendon, Oxford, 1984).

${ }^{42}$ G. L. Squires, Introduction to the Theory of Thermal Neutron Scattering (Dover, New York, 1996).

${ }^{43}$ M. Bée, Quasielastic Neutron Scattering (Adam Hilger, Bristol, 1988).

${ }^{44}$ A. Rahman, K. S. Singwi, and A. Sjölander, Phys. Rev. 126, 986 (1962).

${ }^{45}$ R. Zorn, Phys. Rev. B 55, 6249 (1997).
${ }^{46}$ F. Mezei, Neutron Spin Echo, Lecture Notes in Physics (Springer-Verlag, Heidelberg, 1980), Vol. 28.

${ }^{47}$ B. Farago, Physica B 113, 241 (1997).

${ }^{48}$ Neutron Spin Echo Spectroscopy: Basics, Trends and Applications, edited by F. Mezei, C. Pappas, and T. Gutberlet (Springer, Berlin, 2003).

${ }^{49}$ B. Alefeld, M. Birr, and A. Heidemann, Naturwiss. 56, 410 (1969).

${ }^{50}$ B. Frick and M. Gonzalez, Physica B 301, 8 (2001).

${ }^{51}$ S. W. Bunte and H. Sun, J. Phys. Chem. B 104, 2477 (2000).

${ }^{52}$ H. Sun, J. Phys. Chem. B 102, 7338 (1998).

${ }^{53}$ J. Yang, Y. Ren, A. Tian, and H. Sun, J. Phys. Chem. B 104, 4951 (2000).

${ }^{54}$ D. N. Theodorou and U. W. Suter, Macromolecules 18, 1467 (1985).

${ }^{55}$ Physical Properties of Polymers Handbook, edited by J. E. Mark (Springer, New York, 1996).

${ }^{56}$ J. Colmenero, F. Alvarez, and A. Arbe, Phys. Rev. E 65, 041804 (2002).

${ }^{57}$ R. Zorn, A. Arbe, J. Colmenero, B. Frick, D. Richter, and U. Buchenau, Phys. Rev. E 52, 781 (1995).

${ }^{58}$ A. Chahid, A. Alegría, and J. Colmenero, Macromolecules 27, 3282 (1994).

${ }^{59}$ The amplitudes have been corrected for band-pass effects with help of the simulations using the procedure followed in previous works (Refs. 31, 35 , and 38 ).

${ }^{60}$ S. Mossa, R. Di Leonardo, G. Ruocco, and M. Sampoli, Phys. Rev. E 62, 612 (2000).

${ }^{61}$ A. Soldera and Y. Grohens, Polymer 45, 1307 (2004).

${ }^{62}$ R. Pérez-Aparicio, A. Arbe, F. Alvarez, J. Colmenero, and L. Willner, Macromolecules 42, 8271 (2009).

${ }^{63}$ G. D. Smith, W. Paul, M. Monkenbusch, L. Willner, D. Richter, X. H. Qiu, and M. D. Ediger, Macromolecules 32, 8857 (1999).

${ }^{64}$ M. Tyagi, A. Arbe, A. Alegría, J. Colmenero, and B. Frick, Macromolecules 40, 4568 (2007).

${ }^{65}$ R. Pérez Aparicio, A. Arbe, J. Colmenero, B. Frick, L. Willner, D. Richter, and L. J. Fetters, Macromolecules 39, 1060 (2006).

${ }^{66}$ J. Colmenero, A. J. Moreno, and A. Alegría, Prog. Polym. Sci. 30, 1147 (2005).

${ }^{67}$ F. Alvarez, A. Arbe, and J. Colmenero, Chem. Phys. 261, 47 (2000).

${ }^{68} \mathrm{~F}$. Alvarez et al. (unpublished).

${ }^{69}$ D. Richter, M. Monkenbusch, L. Willner, A. Arbe, J. Colmenero, and B. Farago, Europhys. Lett. 66, 239 (2004).

${ }^{70}$ U. Bengtzelius, W. Götze, and A. Sjölander, J. Phys. C 17, 5915 (1984).

${ }^{71}$ G. F. Signorini, J.-L. Barrat, and M. L. Klein, J. Chem. Phys. 92, 1294 (1990).

${ }^{72}$ M. Aichele and J. Baschnagel, Eur. Phys. J. E 5, 229 (2001).

${ }^{73}$ W. Kob and H. C. Andersen, Phys. Rev. E 51, 4626 (1995).

${ }^{74}$ B. Doliwa and A. Heuer, Phys. Rev. Lett. 80, 4915 (1998).

${ }^{75}$ The $\alpha$-scaling has to be checked by choosing a value for the decay of the correlation function small enough to assure that the relevant process driving the dynamics there is the $\alpha$-relaxation. The resulting quality of the scaling representation does not change if we choose any value below 0.3.

${ }^{76}$ M. Fuchs, J. Non-Cryst. Solids 172-174, 241 (1994).

${ }^{77}$ M. Fuchs, W. Götze, and M. R. Mayr, Phys. Rev. E 58, 3384 (1998).

${ }^{78}$ J. Horbach and W. Kob, Phys. Rev. E 64, 041503 (2001).

${ }^{79}$ F. Sciortino, L. Fabbian, S.-H. Chen, and P. Tartaglia, Phys. Rev. E 56, 5397 (1997).

${ }^{80}$ A. Narros, Ph.D. thesis, Universidad del Pa's Vasco (UPV/EHU), 2007.

${ }^{81}$ F. Sciortino, P. Tartaglia, and E. Zaccarelli, Phys. Rev. Lett. 91, 268301 (2003).

${ }^{82}$ M. Sperl, Phys. Rev. E 68, 031405 (2003).

${ }^{83}$ E. Zaccarelli, G. Foffi, K. A. Dawson, S. V. Buldyrev, F. Sciortino, and P. Tartaglia, Phys. Rev. E 66, 041402 (2002).

${ }^{84}$ A. J. Moreno and J. Colmenero, J. Chem. Phys. 124, 184906 (2006).

${ }^{85}$ A. J. Moreno and J. Colmenero, Phys. Rev. E 74, 021409 (2006).

${ }^{86}$ A. J. Moreno and J. Colmenero, J. Chem. Phys. 125, 164507 (2006).

${ }^{87}$ V. Krakoviack, Phys. Rev. Lett. 94, 065703 (2005). 